\title{
Monte Carlo dosimetry of the eye plaque design used at the St. Erik Eye Hospital for I-125 brachytherapy
}

Mattias Karlsson, Josef Nilsson, Marie Lundell and Åsa Carlsson Tedgren

\author{
Linköping University Post Print
}

\section{Tweet}

N.B.: When citing this work, cite the original article.

Original Publication:

Mattias Karlsson, Josef Nilsson, Marie Lundell and Åsa Carlsson Tedgren, Monte Carlo dosimetry of the eye plaque design used at the St. Erik Eye Hospital for I-125 brachytherapy, 2014, Brachytherapy, (13), 6, 651-656.

http://dx.doi.org/10.1016/j.brachy.2014.05.018

Copyright: Elsevier

http://www.elsevier.com/

Postprint available at: Linköping University Electronic Press

http://urn.kb.se/resolve?urn=urn:nbn:se:liu:diva-112619 


\title{
Monte Carlo dosimetry of the eye plaque design used at
}

\author{
the St. Erik Eye Hospital for ${ }^{125}$ I brachytherapy
}

MATTIAS KARLSSON ${ }^{1,2^{*}}$, JOSEF NILSSON ${ }^{2}$, MARIE LUNDELL $^{2,3}$, ÅSA CARLSSON TEDGREN ${ }^{4,5}$

${ }^{1}$ Department of Medical and Health Sciences, Linköping University, SE-581 85

Linköping, Sweden

${ }^{2}$ Department of Medical Physics, Karolinska University Hospital,

SE-171 76 Stockholm, Sweden

${ }^{3}$ Department of Oncology, Karolinska Institutet, SE-17176 Stockholm

${ }^{4}$ Swedish Radiation Safety Authority, SE-171 16 Stockholm, Sweden

${ }^{5}$ Department of Medical and Health Sciences, Linköping University, and Center for Medical Image Science and Visualization, SE-581 85 Linköping, Sweden

Running title: "Monte Carlo dosimetry for the St. Erik eye plaque"

We disclose that none of the co-authors (Mattias Karlsson, Josef Nilsson, Marie Lundell, Åsa Carlsson Tedgren) have any conflict of interest, of financial or other nature, in the current work.

*Corresponding author: Mattias Karlsson, Radiation Physics, Department of Medical and Health Sciences, Linköping University, SE-581 85 Linköping,

Sweden, Tel.:+46(0)87994485; fax: +46(0)87994010

E-mail address: mattias.karlsson@liu.se 


\section{Abstract}

Purpose: At St. Erik Eye Hospital in Stockholm, ocular tumors of apical height $>6 \mathrm{~mm}$ are treated with brachytherapy, using ${ }^{125}$ I seeds attached to a gold alloy plaque while the treatment planning is performed assuming homogeneous water surroundings. The aim of this work was to investigate the dose modifying effects of the plaque and the seed fixating silicone rubber glue.

Methods and materials: The impact of the gold plaque and the silicone rubber glue was studied with the Monte Carlo code MCNP5.

Results: For the $2 \mathrm{~cm}$ most proximal to the plaque surface along the plaque central axis, the eyeball received $104.6 \%-93.0 \%$ of the dose in all-water conditions.

Conclusions: The $0.3 \mathrm{~mm}$ thick layer of silicone rubber glue, used for seed fixation, attenuates photons little enough to allow characteristic $X$-rays from the gold alloy-plaque to reach the eyeball. Close to the plaque, the dose rates were higher with the plaque and glue present, than in homogeneous water conditions. This is in contrast to what has been reported for more commonly used eye plaques, demonstrating the importance of investigating the dosimetry of individual treatment systems.

Key words: Keywords: Eye plaque; 125I; Dosimetry; Monte Carlo simulations; Brachytherapy; Ocular melanoma. 


\section{Introduction}

Each year about 70-80 persons in Sweden are diagnosed with ocular melanoma. If enucleation of the affected eye can be avoided, the treatment is then brachytherapy, in which a radioactive plaque is attached to the part of sclera adjacent to the underlying ocular tumor. At the St. Erik Eye Hospital (Stockholm, Sweden), tumors with an apical height below $6 \mathrm{~mm}$ are treated with the $\beta$-ray emission from ${ }^{106} \mathrm{Ru}$. The limited range of $\beta$-rays makes them inappropriate for larger tumors due to high proximal sclera doses, where instead discrete ${ }^{125}$ I seeds arranged within a collimating metal plaque are used. The Collaborative Ocular Melanoma Study (COMS) was initiated in 1985 to evaluate the effectiveness of radiotherapy for ocular melanoma. The COMS study of patients with melanomas of apical height between 2.5 and $10.0 \mathrm{~mm}$ found no significant differences in survival rate between patients receiving ${ }^{125}$ | brachytherapy and enucleation (1). However, eye-sparing brachytherapy often retains useful visual acuity and can thus lead to a better life-quality for the patient. COMS has established standardized ${ }^{125} \mathrm{I}$ gold plaques in different sizes, which are today in widespread clinical practice and are the most frequently used plaque models for eye brachytherapy (2).

At St. Erik's, custom-made gold plaques are used for ${ }^{125}$ eye brachytherapy. To the apex of the tumor, a dose of 80 Gy is prescribed, based on TG-43 assumptions. While the COMS system uses silastic seed carriers that establish fixed seed positions and a distinct distance to the sclera, seeds are attached manually to the St. Erik plaque using a thin layer of silicone rubber glue. 
Dosimetry performed according to the dosimetry protocol published by the American Association of Physicists in Medicine (AAPM) Task Group 43 (TG43) $(3,4)$ is based on the dosimetric characteristics for a single seed, derived in a large, homogeneous water volume. Due to the low energy of the photons emitted in the decay of ${ }^{125} \mathrm{I}$ (average $28 \mathrm{keV}$ ), non water-equivalent materials in the vicinity of the seeds may considerably affect the dose distribution. An obvious concern in eye plaque therapy is the metal plaque to which the seeds are attached. The dose modifying effects of backing materials of high atomic number have been investigated in several studies (5-13). For the $20 \mathrm{~mm}$ diameter COMS plaque loaded with ${ }^{125}$ I seeds, the dose reduction is on the order of $13-14 \%$ at $10 \mathrm{~mm}$ depth from inner sclera, compared to TG-43 conditions $(11,14)$. Another plaque design, the OSU-NAG plaque, has similarities with the St. Erik plaque in that the seeds are manually glued instead of being positioned in a seed carrier (as for COMS plaques) $(15,16)$. They differ, however, in that the OSU-NAG plaque displaces the seeds $2 \mathrm{~mm}$ from sclera to avoid high dose regions and to reduce the tumor dose gradient.

To compare treatment outcomes and explain side effects from plaque therapy, accurate dosimetry is essential. The report from AAPM TG-129 (2) states that the resulting dose distributions are highly sensitive to the plaque design and the seed model used. This study was initiated to investigate the impact of the plaque and rubber glue heterogeneity of the St. Erik plaque on the dose distribution. Currently, AAPM TG-221 is reviewing the dosimetry of non-COMS plaques for ocular brachytherapy. The dosimetry of this plaque model has not been studied in detail before. 


\section{Methods and materials}

\section{Plaque and seeds}

The CCB St. Erik plaque investigated in this study was modeled with diameter $20.0 \mathrm{~mm}$, inner height $5.0 \mathrm{~mm}$ and thickness $0.5 \mathrm{~mm}$. The radius of curvature of the concave plaque surface is $11.8 \mathrm{~mm}$. The design of the CCB plaque is shown in Figure 1. It has been custom-made by a goldsmith to replicate a ${ }^{106}$ Ru-plaque of type CCB (Eckert \& Ziegler BEBIG GmbH, Berlin, Germany). In addition to the $\mathrm{CCB}$ plaque, plaques replicating models $\mathrm{CCA}, \mathrm{CCC}, \mathrm{CIB}, \mathrm{COB}$ and CCZ have also been manufactured. The plaque consists of an 18 carat gold alloy. Due to lack of data on the elemental composition, alloy data was taken from the work on gold alloys in jewelry by Raub and Ott (17). The adopted alloy consisted of $75.0 \% \mathrm{Au}, 15.5 \% \mathrm{Ag}, 9.0 \% \mathrm{Cu}$ and $0.5 \% \mathrm{Zn}$ by weight, with a mass density, $\rho=15.4 \mathrm{~g} / \mathrm{cm}^{3}$. The seeds are manually attached to the plaque with rubber glue. As seen in Figure 1, the glue does not fully cover the seeds. The distance between the outer sclera and the proximal protruding seed edge is uncertain. Due to this uncertainty, the origin of the coordinate system was set at the external rubber glue surface on the plaque's central axis (CAX), and not at the inner sclera as in the COMS reference coordinate system (2).

The seeds are arranged in a predetermined pattern specially designed for the CCB plaque, Figure 2. The endpoint coordinates for each seed is given in Table 1 together with the angle $\varphi$, which is the angle between the positive $x$ axis and the line through the center of a seed and the origin, projected on the $x$ - 
$y$ plane. The 10 seeds are arranged with 7 seeds close to the edge of the plaque and 3 seeds in an equilateral triangle close to the center of the plaque. The 7 seeds are positioned $2.0 \mathrm{~mm}$ from the edge of the plaque. They lie on an isocentric circle with circumference $49 \mathrm{~mm}$. Each seeds is $4.5 \mathrm{~mm}$ long and the space between the seeds is therefore $2.5 \mathrm{~mm}$. The 3 seeds in the bottom are positioned with approximately $2 \mathrm{~mm}$ space between the endpoints of two neighboring seeds. Due to the manual application, errors in positions and orientations of the seeds may occur. It is outside the scope of the present paper to investigate positional uncertainties of the St Erik system in detail, but it can be noted that the seed placement procedure is similar to that of the OSUNAG plaque, which is discussed in Zhang et al. (16).

The gluing procedure is to first apply a thin layer over the whole concave plaque surface. The seeds are then manually pressed to the glue. The average glue layer thickness was estimated using a micrometer caliper on 5 randomly chosen glue sheets that had been removed from the plaque after completed treatments. The thicknesses ranged from 0.2 to $0.4 \mathrm{~mm}$, with an average of 0.3 mm. The silicone rubber (RTV 3140, Dow Corning Corp., Midland, MI) has a density of $1.05 \mathrm{~g} / \mathrm{cm}^{3}$ (18) with the molecular formula [SiO2 (CH3)]n (19), yielding an elemental composition of $4.0 \% \mathrm{H}, 16.0 \% \mathrm{C}, 42.6 \% \mathrm{O}$ and $37.4 \% \mathrm{Si}$ by weight.

The ${ }^{125} \mathrm{I}$ seed model used with the plaque is the IsoSeed $\circledast \mathrm{I} 25 . \mathrm{S} 16$ (Eckert \& Ziegler BEBIG GmbH, Berlin, Germany). This model is geometrically identical to the IsoSeed $®$ I25.S06 model (Eckert \& Ziegler BEBIG GmbH, Berlin, Germany), but has higher seed strength. 
Table 1. Positions for each seed in the CCB model of the St. Erik plaque, given with the end coordinates and the angle $\varphi$.

\begin{tabular}{|c|c|c|c|c|c|c|c|}
\hline & \multicolumn{6}{|c|}{ Seed end coordinates [mm] } & \multirow{2}{*}{$\begin{array}{c}\text { Angle } \\
\varphi\left[^{\circ}\right]\end{array}$} \\
\hline Seed \# & $x_{1}$ & $y_{1}$ & $Z_{1}$ & $x_{2}$ & $y_{2}$ & $Z_{2}$ & \\
\hline 1 & -0.482 & -0.586 & 0.320 & -0.064 & -0.769 & 0.320 & 248.1 \\
\hline 2 & 0.183 & -0.751 & 0.320 & 0.574 & -0.517 & 0.320 & 300.8 \\
\hline 3 & 0.749 & -0.268 & 0.320 & 0.762 & 0.188 & 0.320 & 357.0 \\
\hline 4 & 0.645 & 0.415 & 0.320 & 0.316 & 0.731 & 0.320 & 50.0 \\
\hline 5 & -0.008 & 0.776 & 0.320 & -0.431 & 0.604 & 0.320 & 107.7 \\
\hline 6 & -0.575 & 0.509 & 0.320 & -0.773 & 0.099 & 0.320 & 155.7 \\
\hline 7 & -0.774 & -0.037 & 0.320 & -0.618 & -0.465 & 0.320 & 199.8 \\
\hline 8 & -0.100 & -0.309 & 0.070 & 0.308 & -0.105 & 0.070 & 296.6 \\
\hline 9 & 0.327 & 0.019 & 0.070 & -0.014 & 0.322 & 0.070 & 47.5 \\
\hline 10 & -0.238 & 0.236 & 0.070 & -0.225 & -0.219 & 0.070 & 177.8 \\
\hline
\end{tabular}

The angle $\varphi$ is the angle between the positive $x$-axis and a line through origin and the seed center, projected on the $x-y$ plane. The coordinate system is defined with $x=0$, $y=0, z=0$ at the external silicone rubber glue surface on the plaque central axis, as indicated in Figure 1.

\section{Monte Carlo technique}

Monte Carlo calculations of dose distributions were performed with the generalpurpose code MCNP5 version 1.51 (20). All simulations used the default photon cross-section library MCPLIB04, based on ENDF/B-VI.8, which in turn is derived from EPDL97 (21). The decay spectrum for ${ }^{125}$ I was taken from TG$43 \cup 1$ (4). Because of the low energies of photons emitted in the decay of ${ }^{125}$, charged particle equilibrium was assumed in the scoring voxels and absorbed dose was approximated as collision kerma. This justified the use of the energy 
deposition tally (F6) for the scoring of absorbed dose rate and collision kerma in the derivation of TG-43 parameters.

\section{Single seed simulations}

As a first step, a single 125.S16 seed was modeled in MCNP5 and TG-43 parameters were derived. Using the line-source approximation, the values of the radial dose function, $g\llcorner(r)$, anisotropy function, $F(r, \theta)$, air-kerma strength, $S_{k}$, and dose-rate constant, $\Lambda$, were determined.

Dimensions and material compositions were taken from specifications described in the work by Hedtjärn et al. (22).

The seed was positioned in the center of a water sphere of radius $30 \mathrm{~cm}(\rho=$ $0.998 \mathrm{~g} / \mathrm{cm}^{3}$ ) when estimating $g(r)$ and $F(r, \theta)$. When estimating $S_{k}$, the seed was centered in a vacuum sphere of radius $100 \mathrm{~cm}$ and collision kerma was scored in air-filled cylindrical cells with thickness $1.0 \mathrm{~mm}$ and height $5.0 \mathrm{~mm}$.

\section{Multiseed simulations}

To investigate effects of the plaque heterogeneity, two multiseed MC models were created; one with homogeneous water surroundings (homo) and one with added heterogeneities (hetero). The homo model contained only the seeds in representative treatment positions, embedded in water. The hetero simulation also contained the plaque and a layer of $0.3 \mathrm{~mm}$ silicone rubber glue, with the seeds in the same positions as the homo model.

Verification of the homo model was performed by comparing TPS predictions of the CAX dose rate to MC results. The seeds were in the same positions in both 
calculations. Calculations with the clinical TPS BrachyVision 8.9 (Varian Medical Systems, Inc., Palo Alto, CA) were performed according to the TG-43 formalism using TG-43 consensus data (4) for dose-rate constant, anisotropy function and radial dose function. No corrections were made to account for the plaque or the silicone rubber. In the MC model, the seeds were positioned in the center of a homogenous water sphere of radius $30 \mathrm{~cm}$ (homo conditions). Since all seeds were present in the MC model, interseed attenuation was modeled which was not taken into account in the TPS calculations. Interseed effects for different seed models along the CAX of the COMS plaque have been reported to be below 1\% (10).

Additional simulations were performed to estimate the effect on the CAX dose rate when the glue layer thickness in the hetero model was varied from 0.0 to $0.5 \mathrm{~mm}$ in steps of $0.1 \mathrm{~mm}$.

In all multiseed simulations, the CAX dose rate was estimated using the energy deposition tally F6 with 100 cubical scoring cells $\left(0.02 \times 0.02 \times 0.02 \mathrm{~cm}^{3}\right.$ each). Each simulation was run with $6 \times 10^{9}$ starting particles.

Heterogeneity effects were also studied on 3D dose rate distributions. Dose rates were simulated in a rectilinear 3D voxel mesh, superimposed over the geometry, using the FMESH flux tally feature in MCNP5. An FM tally multiplier card was used to transform the FMESH tally to an energy deposition tally, giving the same results as tally F6. The scoring mesh consisted of $40 \times 60 \times 60$ voxels of $0.05 \times 0.05 \times 0.05 \mathrm{~cm}^{3}$ each. Simulations for 3D dose data were run with $2 \times 10^{9}$ starting particles. 
All multiseed simulations were performed with the seeds positioned at the center of a water sphere of radius $30 \mathrm{~cm}\left(\rho=0.998 \mathrm{~g} / \mathrm{cm}^{3}\right)$. The plaque has two suture eyelets which were neglected in the MC model.

\section{Results}

Reported uncertainties are based on the $1 \sigma$ statistical uncertainty from the MC simulations, providing a level of confidence of approximately $68 \%$.

Values for $g(r)$ agreed to TG-43U1 consensus data (or data from candidate datasets) within 2.6\%. $F(r, \theta)$ agreed within $5.0 \%$ at all angles except for the smallest $\theta\left(\leq 5^{\circ}\right)$ and $r(\leq 1.0 \mathrm{~mm})$ where deviations up to $8.8 \%$ were seen. The MC derived value of $\wedge$ had a percentage difference to the TG-43U1 consensus value of $-1.33 \%$.

The normalized CAX dose rates calculated with TPS and with MC in homo conditions agreed within $0.6 \%$.

The ratios of the CAX dose rates, simulated in hetero conditions, to that in homo conditions is shown in Figure 3 . The ratio was at a maximum closest to the plaque $(104.6 \%$ at $z=0.02 \mathrm{~mm})$, decreased to $96.3 \%$ at $z=10.0 \mathrm{~mm}$, and $93.0 \%$ at the most distal point simulated, $z=20.0 \mathrm{~mm}$.

The 2D plot in Figure 4 shows the hetero/homo-ratio in a cross-sectional view through the center of the plaque. The $92 \%$ isoline approximately coincides with the contour of the eyeball, when the plaque is fitted tightly to it.

Scleral doses were estimated based on the simulation results for hetero conditions. When prescribing $80 \mathrm{~Gy}$ to a tumor apex at $7 \mathrm{~mm}$ from the external 
silicone surface, the dose delivered to the apex will be 78.4 Gy (hetero/homoratio $98.0 \%$ ). The dose to a point $1 \mathrm{~mm}$ from the rubber surface (approximately the depth of the inner sclera) will then be $295 \mathrm{~Gy}$. For a tumor apex $12 \mathrm{~mm}$ from the external rubber surface, the delivered dose will be $76.8 \mathrm{~Gy}$ (hetero/homo-ratio 96.0\%) and the dose at $1 \mathrm{~mm}$ distance $675 \mathrm{~Gy}$. The dose to a $1 \mathrm{~mm}^{3}$ cubic voxel centered directly in front of seed 9 (see Figure 2) was estimated to $472 \mathrm{~Gy}-1126 \mathrm{~Gy}$ for prescription points at $7-12 \mathrm{~mm}$ from the rubber surface on the plaque's CAX.

Results of the simulations of the CAX dose rates with various thicknesses of silicone rubber are shown in Figure 5. The results were normalized to the dose rates with a $0.3 \mathrm{~mm}$ thick rubber glue layer. Close to the plaque, the relative dose rates differed by several percent, up to $4.3 \%$ for the bare plaque $(0.0 \mathrm{~mm}$ thickness). The differences decreased with distance. From approximately 3.0 $\mathrm{mm}$ from the external rubber glue surface, the dose rates were within $\pm 1.0 \%$ for all layer thicknesses investigated.

\section{Discussion}

In simulations of 10 seeds attached with $0.3 \mathrm{~mm}$ silicone rubber to the gold plaque, the dose rate close to the plaque exceeded that in homogeneous water conditions, as predicted by TG-43 based TPS. This is in contrast to the dose modifying effects of the more commonly used COMS plaque, for which studies have shown a hetero/homo-ratio consistently below 1.00 , mainly due to attenuation in the silastic seed carrier $(11,14,23)$. The thinner seed fixative used with the St. Erik plaque attenuates less of the primary radiation and the 
characteristic X-rays from the gold alloy backing. Since most TG-43 based TPS do not account for material inhomogeneities, the smaller amounts of fixative result in dose distributions close to TPS predictions. Thus, comparing doseresponse data for different ocular brachytherapy systems in e.g. clinical trials, would hence be more accurate if plaque heterogeneities are taken into account than if based solely on homogenous water doses.

The aim of this study was to investigate the impact of the plaque and the rubber glue to the dose distribution. There are several other factors that may affect the dose distributions or introduce uncertainties. One such factor is the variation in the rubber glue layer thickness that may occur due to manual application. Simulated dose rates along the CAX of the plaque with different layer thicknesses showed variations in dose rates close to the plaque. Tumors treated with ${ }^{125} \mathrm{I}$ at the St. Erik Eye Hospital have an apical height exceeding 6 $\mathrm{mm}$, i.e. with the apex located at least $7 \mathrm{~mm}$ from the plaque surface. At this depth, the inaccuracy due to variations in rubber thickness is not likely to be larger than $1 \%$.

In the assembled plaque, the layer of silicone rubber does not fully cover the seeds and the protruding parts of the sources will be in direct contact with sclera. Other plaque designs, as the COMS plaque and the OSU-Nag plaque, displace the seeds to maintain a certain distance to sclera, to reduce the sclera dose and the tumor dose gradient $(2,15)$. The protruding sources of the St. Erik plaque make the position of the plaque relative to the sclera uncertain. In reality, there might be a sub-millimeter air-layer between them, displacing the plaque by the same distance. In this study, the seeds and plaque were 
immersed in water. Under this assumption, the dose to the inner sclera was estimated with a voxel positioned $1 \mathrm{~mm}$ from the external rubber glue surface on the plaque's CAX. For a prescription dose of $80 \mathrm{~Gy}$ to points located $7-12$ $\mathrm{mm}$ from the external glue surface (i.e. an apical height of $6-11 \mathrm{~mm}$ ), the dose to the point at $1 \mathrm{~mm}$ distance was estimated to $295-675 \mathrm{~Gy}$. To put this in perspective, doses reported by Thomson et al. (14) (as reproduced in the report of TG-129 (2)) for the COMS plaque of $20 \mathrm{~mm}$ diameter, loaded with ${ }^{125}$ | seeds of model OncoSeed 6711 (Oncura, a Unit of GE Healthcare, Chalfont St. Giles, UK) was rescaled. Doses to the inner sclera, for a prescribed dose of 85 Gy in the COMS plaque, were estimated to $232 \mathrm{~Gy}$ and $508 \mathrm{~Gy}$ for tumors with apical heights of $6 \mathrm{~mm}$ and $11 \mathrm{~mm}$ respectively. This is to be seen as a rough comparison since the plaque designs, the source positions, number of sources and source models differ.

\section{Conclusion}

$A^{125}$ eye plaque brachytherapy system for treatment of ocular tumors with apical height larger than $6 \mathrm{~mm}$ was studied by means of MC simulations.

When taking the heterogeneities of the plaque and seed fixating glue into account, dose rates along the plaque central axis exceeded the homogeneous water situation by $4.6 \%$ close to the plaque, but decreased it by $7.0 \%$ at 20.0 $\mathrm{mm}$ from the external glue surface. The high dose ratios close to the plaque were attributed mainly the thin glue layer, which allows characteristic X-rays from the gold alloy backing to reach the eyeball. 
Variations in the silicone rubber thickness that could arise due to the manual application did not affect the central axis dose rates by more than $1 \%$ at depths greater than $3.0 \mathrm{~mm}$ from the external surface of the rubber glue. Close to the plaque, the dose rate was higher in heterogeneous than in homogeneous conditions. This is in contrast to what has been reported for more commonly used eye plaques, demonstrating the importance of investigating the dosimetry of individual systems if outcomes are to be compared in clinical trials or combined in the search for improved dose response data. 


\section{References}

1. Collaborative Ocular Melanoma Study Group. The COMS randomized trial of iodine 125 brachytherapy for choroidal melanoma: V. Twelve-year mortality rates and prognostic factors: COMS report No. 28. Arch Ophthalmol. 2006;124:1684-1693.

2. Chiu-Tsao ST, Astrahan MA, Finger PT, et al. Dosimetry of ${ }^{125}$ and ${ }^{103} \mathrm{Pd}$ COMS eye plaques for intraocular tumors: report of Task Group 129 by the AAPM and ABS. Med Phys 2012;39:6161-6184.

3. Nath R, Anderson LL, Luxton G, et al. Dosimetry of interstitial brachytherapy sources: recommendations of the AAPM Radiation Therapy Committee Task Group No. 43. American Association of Physicists in Medicine. Med Phys 1995;22:209-234.

4. Rivard MJ, Coursey BM, DeWerd LA, et al. Update of AAPM Task Group No. 43 Report: A revised AAPM protocol for brachytherapy dose calculations. Med Phys 2004;31:633-674.

5. Chiu-Tsao ST, Anderson LL, O'Brien K, et al. Dosimetry for ${ }^{125}$ I seed (model 6711) in eye plaques. Med Phys 1993;20:383-389.

6. Astrahan MA, Luxton G, Pu Q, et al. Conformal episcleral plaque therapy. Int J Radiat Oncol 1997;39:505-519. 
7. Krintz AL, Hanson WF, Ibbott GS, et al. A reanalysis of the Collaborative Ocular Melanoma Study Medium Tumor Trial eye plaque dosimetry. Int J Radiat Oncol Biol Phys 2003;56:889-898.

8. Astrahan MA. Improved treatment planning for COMS eye plaques. Int $\mathrm{J}$ Radiat Oncol Biol Phys 2005;61:1227-1242.

9. Granero D, Pérez-Calatayud J, Ballester F, et al. Dosimetric study of the 15 mm ROPES eye plaque. Med Phys 2004;31:3330-3336.

10. Thomson RM, Rogers DWO. Monte Carlo dosimetry for ${ }^{125} \mathrm{I}$ and ${ }^{103} \mathrm{Pd}$ eye plaque brachytherapy with various seed models. Med Phys; 2010;37:368-376.

11. Melhus CS, Rivard MJ. COMS eye plaque brachytherapy dosimetry simulations for ${ }^{103} \mathrm{Pd},{ }^{125}$, and ${ }^{131} \mathrm{Cs}$. Med Phys 2008;35:3364-3371.

12. Chiu-Tsao ST, O'Brien K, Sanna R, et al. Monte Carlo dosimetry for ${ }^{125}$ and ${ }^{60} \mathrm{Co}$ in eye plaque therapy. Med Phys 1986;13:678-682.

13. de la Zerda A, Chiu-Tsao ST, Lin J, et al. ${ }^{125}$ I eye plaque dose distribution including penumbra characteristics. Med Phys 1996;23:407-418.

14. Thomson RM, Taylor REP, Rogers DWO. Monte Carlo dosimetry for ${ }^{125}$ and ${ }^{103}$ Pd eye plaque brachytherapy. Med Phys 2008;35:5530-5543.

15. Nag S, Wang D, Wu H, et al. Custom-made "Nag" eye plaques for ${ }^{125}$ brachytherapy. Int J Radiat Oncol Biol Phys 2003;56:1373-1380. 
16. Zhang H, Davidorf F, Qi Y. Comparison of $16 \mathrm{~mm}$ OSU-Nag and COMS eye plaques. J Appl Clin Med Phys 2012;13:166-178.

17. Raub CJ, Ott D. Gold casting alloys. Gold Bull 1983;16:46-51.

18. Dow Corning Corporation. Manual Safety Data Sheet - DOW CORNING ${ }^{8} 3140$ RTV COATING. 2013 Available at: http://www4.dowcorning.com/DataFiles/090007b281c7cea9.pdf. Accessed May 272013.

19. Nwachukwu FA, Baron MG. Polymeric matrices for immobilising zinc tetraphenylporphyrin in absorbance based gas sensors. Sensors Actuators B Chem 200;90:276-285.

20. X-5 Monte Carlo Team. MCNP - A general Monte Carlo N-particle transport code, Version 5. Los Alamos Natl Lab Rep LA-UR-03-1987. 2003.

21. Cullen DE, Hubbell JH, Kissel L. EPDL97: the evaluated photo data library `97 version. UCRL-50400, Vol. 6, Rev. 5; 1997. Lawrence Livermore National Laboratory.

22. Hedtjärn H, Carlsson G, Williamson J. Monte Carlo-aided dosimetry of the symmetra model I25.S06 ${ }^{125}$ I, interstitial brachytherapy seed. Med Phys 2000;27:1076-1085.

23. Chiu-Tsao ST, Anderson LL, O'Brien K, et al. Dosimetry for ${ }^{125}$ I seed (model 6711) in eye. Med Phys 1993;20:383-389. 


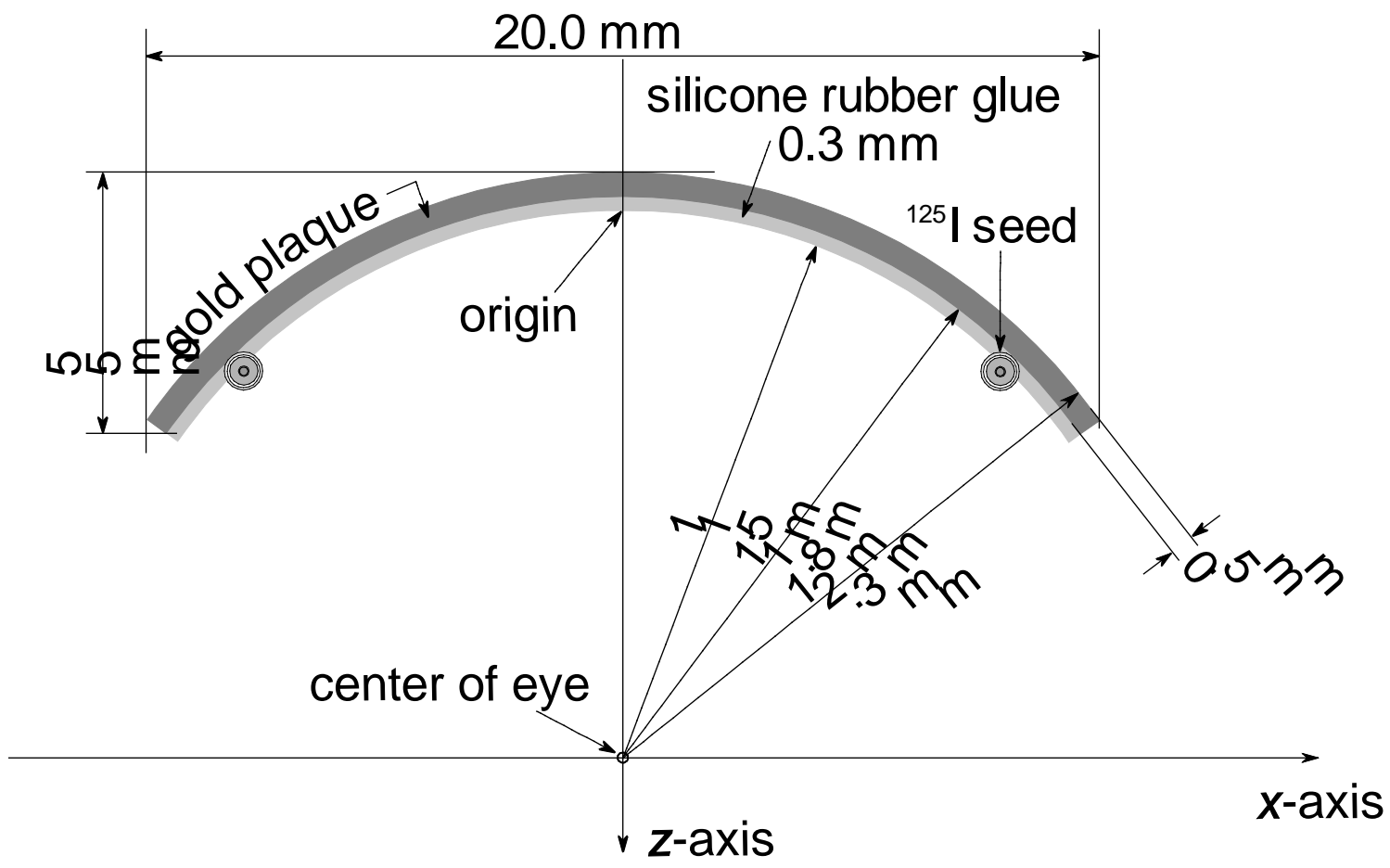

Figure 1

A cross-sectional drawing of the CCB St. Erik plaque, showing the gold backing, the silicone rubber layer and two peripheral $125 \mathrm{I}$ seed. The origin is defined at the external rubber glue surface on the plaque's CAX. The seeds are included to show how they protrude from the glue layer. 


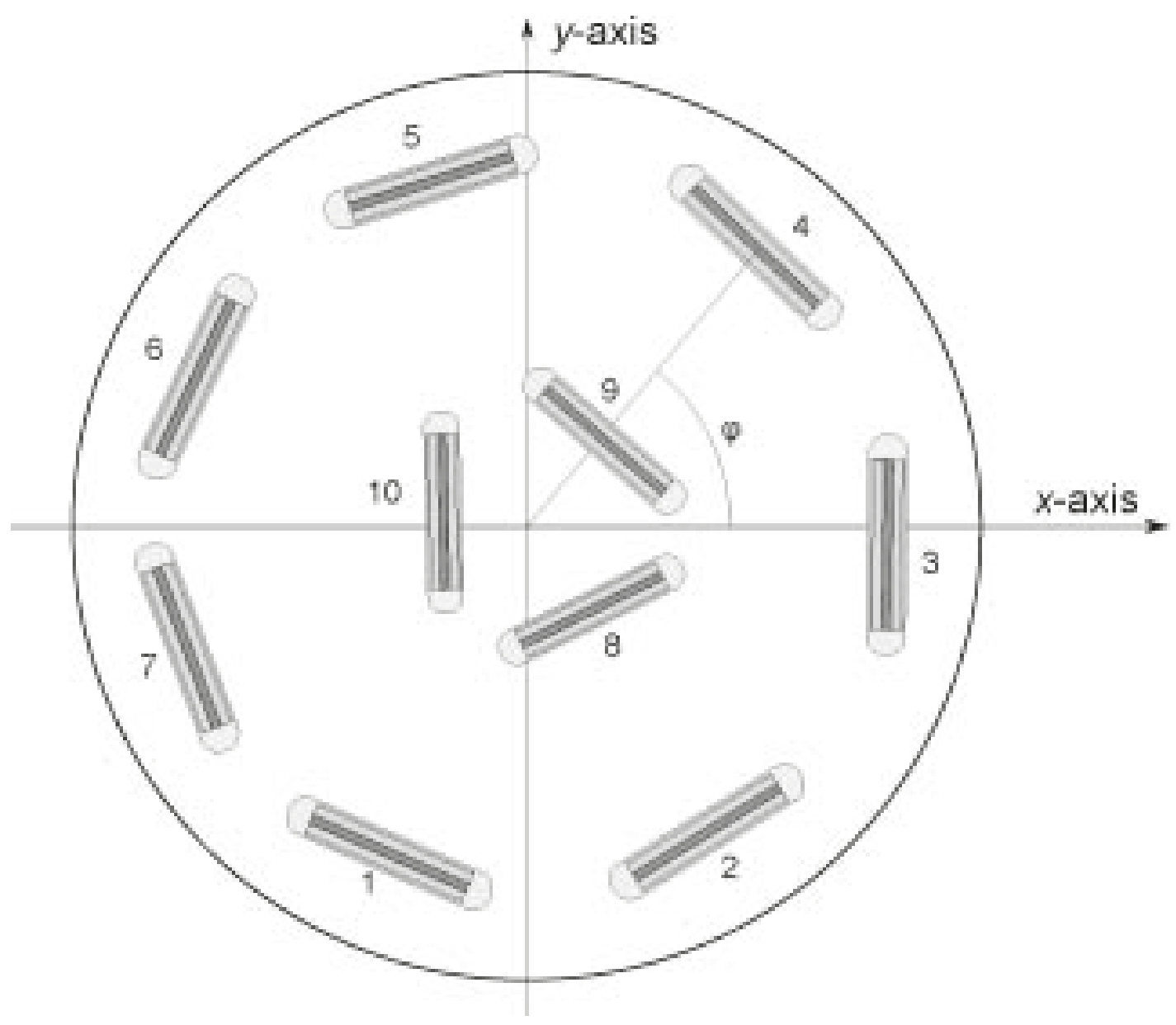

Figure 2

Drawing of the seed positions in the $20.0 \mathrm{~mm}$ diameter CCB plaque. The figure also shows the angle $\varphi$, which is used in Table 1 along with the coordinates of each seed. 


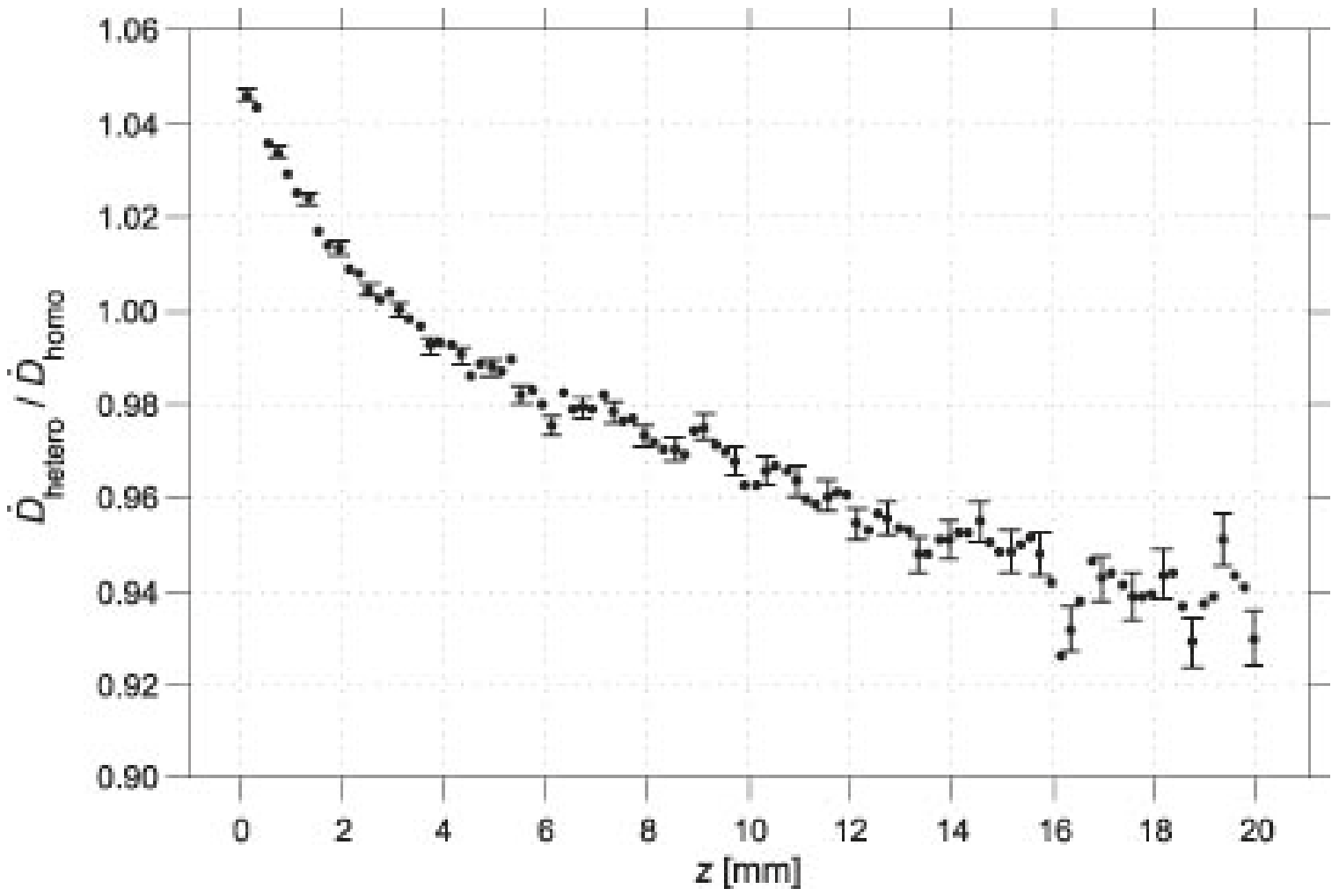

Figure 3

The figure presents the ratio of the dose rates along the CAX of the plaque with the gold alloy backing and rubber glue (hetero) to the dose rate in homogeneous water conditions (homo). 


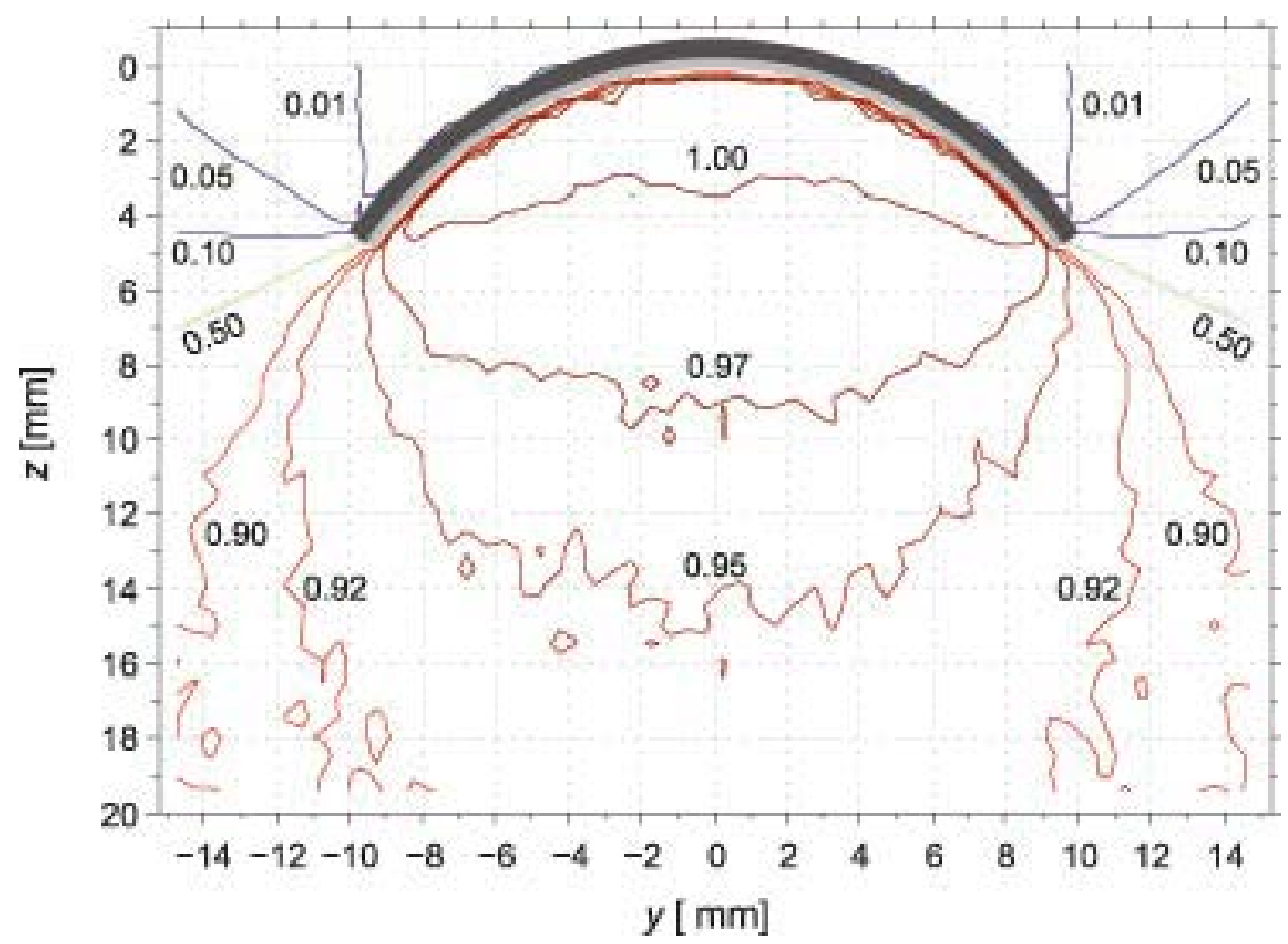

Figure 4

A cross-sectional view through the center of the plaque of the hetero/homoratio, i.e. the ratio of dose rate with gold plaque and rubber present, to the dose rate in homogeneous water conditions. The positions of the plaque (dark gray) and the silicone rubber layer (light gray) have been indicated for guidance 


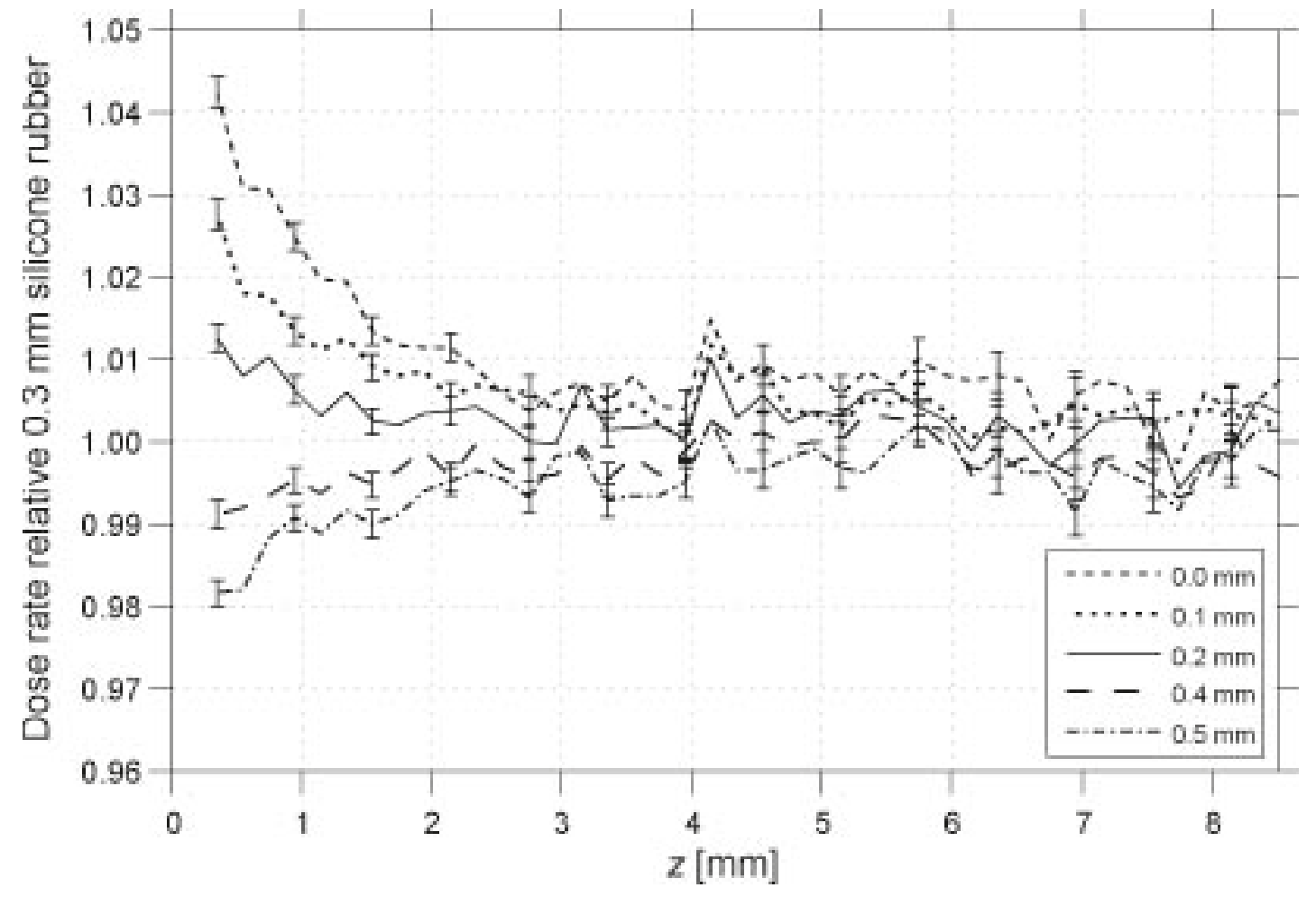

Figure 5

MC simulated dose rates along the CAX of the plaque, for varying thicknesses of the silicone rubber layer. The dose rates are normalized to the dose rate for $0.3 \mathrm{~mm}$ thickness. 